\title{
Future challenges for the treatment of liver tumors
}

\section{Isidoro Di Carlo*1 \& Adriana Toro²}

'Department of Surgical Sciences, Organ Transplantation \& Advanced Technologies, University of Catania, Cannizzaro Hospital, Via Messina 829, 95126 Catania, Italy

2Department of Surgery, Mazzarino S Stefano Hospital, Caltanissetta, Italy

*Author for correspondence: Tel. : +390957264863 = Fax: +390957263020 w idicarlo@unict.it

\section{2nd World Congress of the International Association of Surgeons, Gastroenterologists and Oncologists}

\section{Bangkok, Thailand, 5-8 December 2012}

The annual World Congress of the International Association of Surgeons, Gastroenterologists and Oncologists (IASGO) was held in Bangkok, Thailand, from 5-9 December 2012, hosted under the auspices of the President of the Association, Masatoshi Makuuchi and the General Secretary Nicolas Lygidakis. The President of the Congress and of the local committee was Nopadol Wora-Urai from Bangkok University (Bangkok, Thailand). The organization of the congress was excellent, and both the location and the kindness of the local people were marvellous. The congress was supported by 665 participants from 62 countries, including speakers from Europe, America, Africa, Asia and Australia. The program included symposia, videos, free papers and poster sessions. The scientific sessions also provided live surgery and lunch lectures. The congress offered the opportunity to exchange knowledge about the challenges and controversies involved in the management of abdominal diseases. This paper analyzes current possibilities and future expectations for the diagnosis and treatment of liver tumors, especially hepatocellular carcinomas and liver metastases from colorectal cancer.

N Kokudo (University of Tokyo, Tokyo, Japan) presented a new application of indocyanine green for the intraoperative identification of liver cancer and liver segments for resection. Indocyanine green $(0.5 \mathrm{mg} / \mathrm{kg})$ was administered intravenously for routine preoperative liver function testing, allowing liver tumors to be identified during surgery by fluorescence imaging, through visualization of the indocyanine green retained in differentiated hepatocellular carcinoma (HCC) tissues and noncancerous liver parenchyma compressed by HCC, or liver metastasis [1].

T Beppu (Kumamoto University, Kumamoto, Japan) demonstrated the use of hepatic arterial infusion (HAI) chemotherapy for HCC with vascular invasion. This study, conducted from 1997 to 2007, included 38 HCC patients with macroscopic vascular invasion in the postoperative HAI group, and 35 patients without HAI as the control group. HAI was administered via an implanted injection port and included three courses of weekly cisplatin (daily, $10 \mathrm{mg}$ on days 1-5) and subsequent infusion of 5-fluorouracil (daily, $250 \mathrm{mg}$ on days 1-5). From 2007 , cisplatin $\left(60 \mathrm{mg} / \mathrm{m}^{2}\right)$, 5-fluorouracil $\left(600 \mathrm{mg} / \mathrm{m}^{2}\right)$ and a mixture of mitomycin C $\left(3 \mathrm{mg} / \mathrm{m}^{2}\right)$ and degradable starch microspheres were administered for two courses without the port. The 5-year disease-free survivals were $33.1 \%$ in the HAI group and $11.8 \%$ in the control group $(p=0.029)$. Cox proportional multivariate analysis revealed that HAI was an independent favorable prognostic factor for diseasefree survival for all 73 patients (hazard ratio: $0.536 ; \mathrm{p}=0.029)$. The 5-year overall survival rates were $46.7 \%$ in the HAI group and $32.7 \%$ in the control group ( $p=0.318)$. Adjuvant HAI could, therefore, reduce the recurrence risk in HCC patients with vascular invasion.

M Shimada (Tokushima University, Tokushima, Japan) presented a new anatomical resection of the liver based on the hybrid concept of portal perfusion of the anterior segment and venous drainage of the superior right hepatic vein (SRHV). This technique was based on detailed 3D imaging revealing that the portal perfusion area on the cranial side of the anterior segment sometimes surrounded the SRHV.

The study was based on 3D-computed tomography images of 66 patients with no tumors in the right lobe, constructed using Synapse Vincent software (Fujifilm Corp., Tokyo, Japan). SRHV involvement was defined as involvement of $>1 \mathrm{~cm}$ of the proximal site (near the root) of the SRHV surrounded by the portal perfusion area of the anterior segment. The procedures were: encircling of anterior Glissonian
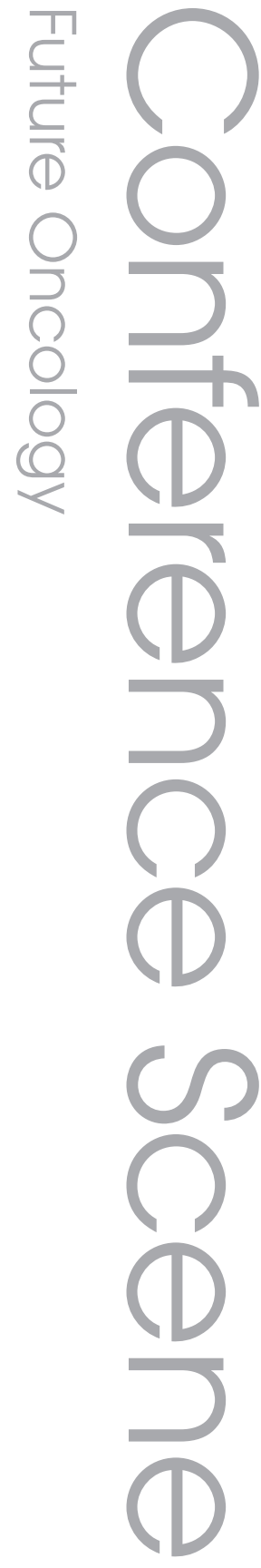

\section{Keywords}

- hepatocellular carcinoma

- laparoscopic surgery = liver metastases $=$ prognosis - survival

\section{Future Medicine part of}


pedicle, SRHV and inferior right hepatic vein (IRHV); confirmation of the demarcation line of the anterior segment by occluding the Glissonian pedicle and demarcation (congested) line by clamping the proper hepatic artery and SRHV; and IRHV-preserved complete resection of the portal perfusion area plus drainage area of SRHV, combined with SRHV resection. SRHV involvement was observed in 17 out of 66 patients $(26 \%)$. A large IRHV (>5-mm diameter) was found in 16 out of 66 patients $(24 \%)$. Large IRHVs were more common in patients with SRHV involvement (eight out of 17; 48\%), compared with those without SRHV involvement (eight out of $49 ; 16 \%$ ). Two patients with large IRHVs and HCC near the SRHV root underwent IRHV-preserved hepatectomy combined with SRHV resection (segment 8 of the liver + SRHV drainage area in one and anterior segment + SRHV drainage area in one). Postoperative computed tomography revealed complete resection of the SRHV drainage area and no congestion in the remnant posterior segment after hepatectomy owing to excellent drainage through a large IRHV.

SRHV involvement was not uncommon, and half of those with SRHV inclusion had a large IRHV. In these HCC patients with a large IRHV, this new hepatectomy technique based on the hybrid concept of portal perfusion of the anterior segment and venous drainage area of the SRHV, combined with SRHV resection, represents a promising option from the viewpoint of systematic resection (curability) and functional reserve of the remnant liver [2].

K Hasegawa (Tokyo University, Tokyo, Japan) presented on a very rare situation involving resection of extrahepatic metastases of HCC limited to the lung and lymph nodes. The surgical indications were three or fewer nodules and single or few nodes from lung and lymph node metastases, respectively. The author performed surgical resections of lung and lymph node metastases in 45 and 16 cases, with postoperative 3-year overall survival rates of 44 and $37 \%$, respectively. Thirteen and four patients experienced long-term survival ( $>3$ years) after resection for lung and lymph node diseases, respectively. Surgical resection for extrahepatic metastases from HCC can have an acceptable prognosis in limited cases, in the event of absent or limited intrahepatic disease [3].

Bleeding during surgery is a serious problem. $S$ Kawamoto (Fukuoka Tokushukai Medical Center, Fukuoka, Japan) investigated this problem in patients with religious objections who underwent hepatic surgery. Appropriate control of back-flow bleeding from hepatic veins increased the safety of hepatic resection during total or selective vascular exclusion for hepatic resection in Jehovah's Witnesses, who cannot accept allogenic blood transfusion. This hepatic resection technique involved: isolation and taping of the left-middle hepatic vein, resection of the short hepatic vein and taping of the right hepatic vein; taping of the infrahepatic inferior vena cava, head-down position, tidal volume less than $300 \mathrm{ml}$; and tourniquet occlusion of the hepatic vein for $1 \mathrm{~min}$ after Pringle clamping. The resection plane of the parenchyma was directed by the hanging maneuver. In the past 12 years, 11 lobectomies and 27 segment/partial hepatectomies were performed for different tumors in Jehovah's Witnesses.

Hemodilution and/or cell-salvaged autologous blood transfusion were applied accordingly. Mean blood loss during the procedure was $410-1522 \mathrm{ml}$. All these resections were performed without transfusion.

N Kokudo demonstrated that aggressive liver resection for liver metastases from colorectal cancer (CRLM) continues to be an option in the molecular-targeted therapy era. This study involved 371 patients who underwent up-front curative surgery for CRLM over a 16-year period, including 273 cases with one to four metastases, and 98 with five or more metastases. Median survival was 7.19 years in the first group and 2.72 years in the second group. Many trials validated the efficacy of neoadjuvant, adjuvant and perioperative chemotherapies for unresectable CRLM, and these approaches have rendered technically resectable CRLM that are not considered suitable for any other treatment resectable. To provide a potentially curative option for patients with unresectable CRLM, there is a clear need for optimal induction chemotherapy, such as the combination of oxaliplatin or irinotecan-based regimens with biologic agents to yield high tumor response rates and conversion to resectability. KRAS status determination is key to selecting the optimal treatment and multidisciplinary management of patients from the time of diagnosis. Current recommendations include an anti-EGF receptor agent, cetuximab or panitumumab, in patients with wild-type $K R A S$ status, and an anti-VEGF agent, bevacizumab, for mutant $K R A S$ status. These methods can reverse the unresectability of CRLM, thus maximizing survival in these patients [4].

JK Sicklick (University of California, CA, USA) presented the role of Hedgehog $(\mathrm{Hh})$ signaling in 
HCC and cirrhosis. The author discussed the possibilities of new drugs for the treatment of HCC. Insights from studies of fetal development and cell regeneration suggest that the abnormal cell growth observed in many malignancy may arise from progenitor cells or tumor stem cells, as a result of disruption of signaling pathways normally required for organ patterning, cell turnover and repair of injured tissues. Hh signaling represents one such pathway. The Hh pathway is critical for normal embryonic development of visceral organs, including the liver. Work by the author's group and others demonstrated that dysregulation and mutation of this signaling pathway are important factors in the progression of HCC, as well as in the genesis of hepatic fibrosis/cirrhosis caused by the proliferation and activation of hepatic stellate cells. Hh signaling represents a potential drug target in both these diseases, while expression of $\mathrm{Hh}$ components has been shown to correlate with prognosis in HCC. AntiHh-targeted therapies are currently being studied in patients with other solid gastrointestinal malignancies, and the potential applications of such drugs for the treatment of both HCC and cirrhosis appear to be promising [5].

\section{References}

1. Kawaguchi Y, Ishizawa T, Miyata Y, et al. Portal uptake function in veno-occlusive regions evaluated by real-time fluorescent imaging using indocyanine green. J. Hepatol. 58(2), 247-253 (2013).

2. Imura S, Shimada M, Miyake K, Ikemoto T, Morine Y, Yoshizumi T. A new technique to acquire additional liver volume for left lobe graft in living donor liver transplantation. Hepatogastroenterology 55, 1206-1210 (2008).
HS Han (Bundang Seoul University, Seoul, South Korea) presented the results of laparoscopic and open surgery for HCC. There have been numerous reports on laparoscopic liver resection for HCC, many of which found laparoscopic surgery to be equivalent to open surgery in terms of survival. However, the application of this procedure is limited when the tumor is located in a postero-superior position, including segments $1,7,8$ and the superior part of segment 4 . However, the development of operative techniques and introduction of new devices means that laparoscopic resection in these difficult locations can be attempted cautiously [6].

\section{Financial \& competing interests disclosure \\ The authors have no relevant affliations or financial involvement with any organization or entity with a financial interest in or financial conflict with the sub- ject matter or materials discussed in the manuscript. This includes employment, consultancies, honoraria, stock ownership or options, expert testimony, grants or patents received or pending, or royalties. \\ No writing assistance was utilized in the production of this manuscript.}

3. Satou S, Ishizawa $\mathrm{T}$, Masuda $\mathrm{K}$ et al. Indocyanine green fluorescent imaging for detecting extrahepatic metastasis of hepatocellular carcinoma. J. Gastroenterol. doi:10.1007/s00535-012-0709-6 (2012) (Epub ahead of print).

4. Saiura A, Yamamoto J, Hasegawa K, et al. Liver resection for multiple colorectal liver metastases with surgery up-front approach: bi-institutional analysis of 736 consecutive cases. World J. Surg. 36, 2171-2178 (2012).
5. Choi SS, Omenetti A, Witek RP et al. Hedgehog pathway activation and epithelialto-mesenchymal transitions during myofibroblastic transformation of rat hepatic cells in culture and cirrhosis. Am. J. Physiol. Gastrointest. Liver Physiol. 297, 1093-1106 (2009).

6. Yoon YS, Han HS, Cho JY, Yoon CJ, Kim JH. Laparoscopic approach for treatment of multiple hepatocellular carcinomas. Surg. Endosc. 26, 3133-3140 (2012). 